\title{
Enhancement of Gratitude in the Context of Elite Athletes: Outcomes and Challenges
}

Kerry Howells and Noleine Fitzallen

University of Tasmania

Accepted manuscript published by Taylor and Francis available at https://doi.org/10.1080/2159676X.2019.1679868

Howells, K., \& Fitzallen. N. (2019). Enhancement of gratitude in the context of elite athletes: Outcomes and challenges. Qualitative Research in Sport, Exercise and Health. [Published online first] doi10.1080/2159676X.2019.1679868

Corresponding author: Dr Kerry Howells (Kerry.Howells@utas.edu.au) 


\title{
Enhancement of Gratitude in the Context of Elite Athletes: Outcomes and Challenges
}

\author{
Kerry Howells and Noleine Fitzallen \\ University of Tasmania
}

\begin{abstract}
Gratitude has been heralded as important to emotional, social and societal wellbeing. A burgeoning body of recent research on the positive impact of gratitude in sport calls for an investigation into its relevance to the field of elite sport, particularly for building resilience and enhancing relationships. An interpretivist/constructivist research approach was adopted to explore the practices, outcomes and challenges of gratitude of ten Australian elite athletesOlympians and Para-Olympians-who participated in a workshop on gratitude. Sports represented in this cohort included cycling, sailing, track and field, beach volleyball and diving. Semi-structured interviews were used to generate data, which were explored using content analysis. Enhancement of gratitude with the elite athletes resulted in the adoption of more meaningful and authentic gratitude towards others. Positive impact of gratitude expressed in this way was reported in the areas of improved attitude; greater awareness; better capacity to deal with stress; increased confidence to express gratitude; and enhanced performance. The paper also discusses the perceived challenges of expressing gratitude within this context.
\end{abstract}

Keywords: stress; resilience; coach-athlete relationships; motivation; positive psychology; gratitude; holistic development

In recent years, a growing body of research has drawn attention to the benefits of gratitude for personal health and wellbeing (e.g., Lavelock et al. 2016, Yoshimura and Berzins 2017). Studies have shown that a grateful disposition offers some protection against depression and anxiety (McCullough, Emmons, and Tsang 2002, Petrocchi and Couyoumdjian 2016), improves sleep (Alkozei et al. 2017, Emmons and McCullough 2003, Ng and Wong 2013), reduces stress, and buffers the effects of traumatic events (Vieselmeyer, Holguin, and Mezulis 2016, Wood, Joseph, and Maltby 2009). 
Researchers in the field of positive psychology also report on the prosocial effects of expressing gratitude (for a recent meta-analytic review see Ma, Tunney, and Ferguson 2017). More specifically, empirical studies have confirmed that there is a correlation between gratitude and prosocial states, such as empathy, forgiveness, generosity, trust, emotional support and willingness to help others (Bartlett and Desteno 2006, McCullough, Emonns, and Tsang 2002, Yost-Dubrow and Dunham 2018). Further, numerous studies have highlighted the important role that gratitude plays in building and maintaining healthy relationships (e.g., Algoe 2012, Algoe, Haidt, and Gable 2008, O’Connell, O’Shea and Gallagher 2016).

The theoretical framework underpinning the concept of gratitude is drawn from a multi-disciplinary perspective (Howells 2012) rather than being embedded purely in psychology. It is acknowledged that gratitude is a complex term that permeates not only our individual disposition but also the way we interrelate with others. Both are influenced by context, including socio-cultural influences. Defined here as the art of giving back out of acknowledgement for what has been received (Howells 2012), gratitude is thus a highly relational construct. Where it has been shown to enhance both personal and social wellbeing in a range of contexts, it is not only wellbeing that is the goal here. For example, within the field of education, Author's work has demonstrated the place gratitude has within the teacher-student relationship (Howells 2012, 2014a, 2014b, 2015). Outcomes of this research show that gratitude can enhance wellbeing of both student and teacher, improve relationships at both an individual and systems level, and improve learning outcomes.

These goals are also highly relevant in the context of elite sport where there is increasing recognition of the importance of coach-athlete relationships to athlete development, performance outcomes, and general wellbeing (e.g., Annerstedt and 
Lindgren 2014, Grahn 2014, Hodge, Graham, and Smith 2014). The positive role that gratitude plays in building and maintaining relationships may also contribute to the development of team cohesion, which is associated with a range of positive outcomes for sporting participants but may involve particular challenges at elite levels of competition (Benson et al. 2016, Filho et al. 2014). In addition, the psychologically protective elements of gratitude may well address some of the challenges reportedly experienced by elite athletes, such as depression and anxiety (Doherty, Hannigan, and Campbell 2016, Nixdorf, Frank, and Beckmann 2016), stress and burnout (Gustafsson, Sagar, and Stenling 2017, Hodge, Lonsdale, and Ng 2008), eating disorders (Giel et al. 2016, Martinsen and Sundgot-Borgen 2013), and substance abuse (Morente-Sánchez and Zabala 2013, Reardon and Creado 2014).

Thus, there are numerous ways in which gratitude-based interventions may be of benefit in the context of elite sport. However, research on the role of gratitude in sport is in its infancy with only a few papers published in this context. An extensive search of the literature found just one in the context of elite sport (Chen and Chang 2014). The present study aims to contribute to addressing this gap in the literature by reporting on the outcomes and challenges of a gratitude intervention that was conducted with a select group of Australian elite athletes.

In acknowledging the complexity in categorising an athlete 'elite', we take the definition from the criteria developed by Swann, Moran and Piggott (2015). All the athletes in our study satisfied the three themes identified as qualifying to be elite: athlete's highest standard of performance in terms of them being among the best in the country at that sport; success at athlete's highest level; and experience at athlete's highest level. Throughout this paper we are using the notion of 'elite' with these criteria in mind. 


\section{Literature Review}

To date, published research on gratitude in the context of sport has employed quantitative methods to investigate associations between gratitude and positive outcomes for athletes. Much of this research has focussed on dispositional gratitude, which has been defined as an affective trait or 'generalized tendency to recognize and respond with grateful emotion to the roles of other people's benevolence in the positive experiences and outcomes that one obtains’ (McCullough, Emmons, and Tsang 2002, 112). In a series of studies, Chen and colleagues investigated associations between dispositional gratitude and measures of wellbeing in athletes. In the first of these studies, Chen and Kee (2008) focused on adolescent athletes and found that dispositional gratitude had a positive impact on team satisfaction and life satisfaction and was negatively associated with athlete burnout. However, a second phase of the study revealed a stronger positive effect for sports-domain gratitude. Building on this, Chen and Chang (2017) investigated the effects of sports-domain gratitude on general wellbeing (as indicated by life satisfaction, vitality, and self-esteem) and sports-specific wellbeing (as indicated by team satisfaction, and low athlete burnout) in athletes in the United States and Taiwan. The study found that the positive effects of sports-domain gratitude held across both samples and further, the positive effects for sports-specific wellbeing were greater than those for general wellbeing. These findings highlight the need for contextualised interpretations of gratitude. Chen and Chang (2017) suggest, 'People live in specific contexts, experience contextual emotions and operate on contextual cognition and behavior, which might not be accurately captured by domaingeneral constructs’ (657-658). 
Existing research on gratitude in sport also lends support to conceptualizations of gratitude as a highly relational construct. For example, Chen (2013) found that the relationship between gratitude and wellbeing in high school athletes was partially mediated by perceived social support from coaches and teammates, while Chen and $\mathrm{Wu}$ (2014) demonstrated that adolescent athletes with high levels of dispositional gratitude increased their self-esteem if they had high levels of affective trust in their coaches. In addition, a recent study of college student athletes in the United States found that relationships among gratitude, burnout, and sports satisfaction were mediated by perceived social support (Gabana et al. 2017).

As noted previously, only one study thus far has focussed on gratitude in the context of elite athletes. Chen and Chang (2014) investigated the directionality between dispositional gratitude and burnout among a group of elite adolescent athletes in Taiwan. In contrast to previous findings by Chen and Kee (2008), the study found that dispositional gratitude had no significant effect on burnout. Rather, athletes’ experiences of burnout had a negative impact on dispositional gratitude and this effect increased over time. One explanation offered by the authors for this result is that in the context of elite sport, athletes' experiences of burnout may be 'more stable and pervasive' (472) than their tendency toward gratitude. This suggests that there may be particular challenges for gratitude-based interventions within the context of elite sport. Indeed, recent qualitative research points to the dominance of performance narratives among elite athletes, which prioritise achievement and winning over alternative measures of success, such as healthy relationships and personal wellbeing (Carless and Douglas 2012, Douglas and Carless 2009). Such a culture would seem to be at odds with holistic approaches to athlete development that place emphasis on sustainability 
and the long-term health of athletes (Barker et al. 2014, Bergeron et al. 2015, Grahn 2014).

In sum, existing research in this area highlights the benefits of gratitude for athletes' wellbeing, but also draws attention to the complexity of the construct and the importance of understanding how it functions within specific contexts. As such, we argue that there is a need for qualitative research that provides a rich account of how gratitude is experienced by elite athletes in the contexts of their personal lives, competition, and training. In addition, we argue that there is a need for research that explores the impact of gratitude as intentional activity as distinct from felt or dispositional gratitude. As noted previously, existing research to date has focussed on the role of dispositional gratitude in sport and little is known about the impact of interventions to induce expressions of gratitude within this context.

Accordingly, the present study investigates the outcomes of an intervention in which gratitude was purposefully expressed by a group of elite athletes. The underlying framework of gratitude used in the intervention is that it is an attitude that is opposite to resentment (Roberts 2004); a state of being that can be prepared for in advance of communicating with others (Howells 2012); and is expressed to others in ways that are meaningful and authentic (Howells 2017; Visser 2009). As indicated by the title of this paper, the notion of enhancement is integral to this research, which proceeds from the assumption that gratitude is essential to everyday interactions and relationships, and in this context, to be an elite athlete. A major purpose of the study is to detail the perceived challenges of expressing gratitude within this context as we argue that these need to be considered in any future gratitude interventions and can further enrich the current literature in the wider field of gratitude research. Our research question is therefore: 
What is the impact of a greater focus on gratitude for elite athletes and what challenges do they perceive in the expression of gratitude?

\section{Research Approach}

In line with research that seeks detailed understanding of participants' perspectives about social phenomena, an interpretivist/constructivist research approach (Creswell 2014) was adopted. The ontological position for this research is constructivist, identifying reality as informed and constructed by ideas, thoughts, and interaction (O'Hara et al. 2011); therefore, recognising participants form their ideas of reality from their own experiences and perceptions. Hence, an interpretive and transactional epistemological framework, encompassing knowledge as a construct informed by experiences, informs this study (Lodico, Spaulding, and Voegtle 2010). While the paradigm of this study may be regarded as a limitation by realists for lacking objectivity gained by a single truth (O'Hara et al. 2011), it does allow for a variety of complex personal truths; thus, recognising the subjectivity of the phenomenon of gratitude and the multiple meanings that arise from individual lived experiences. The interpretivist/constructivist researcher focuses upon the participants' views of the situation being studied (Creswell 2014). Selecting an interpretive paradigm allows the researcher to interpret how the participants are trying to make sense of their experiences. It also facilitates examining the detailed experience of each participant before moving to more general claims. This research approach was deemed suitable because it embraces a complexity of truths to highlight the meaning and practices of gratitude within the lived experience of these elite athletes. The design of this research is experiential in that it places the experience of the participant at the centre to provide insight and understanding within a particular context, rather than take experiences and syphon them down into a standard measure (Stake 2010). Our study is aims to explore 
the experience of the elite athletes, with the purpose of uncovering the impact of their chosen gratitude practices. Interpretivist/constructivist studies are suited to small sample sizes, thus enabling examination of the participants' experiences and perceptions.

Recent studies in sport and exercise psychology have demonstrated that qualitative research approaches can provide valuable insights into the social construction of a range of issues affecting elite athletes, such as eating disorders (Busanich, McGannon, and Schinke 2016), stress related growth (Galli and Reel 2012), parental involvement (Knight et al. 2016) and risk taking (Jones et al. 2017). Prominent within these studies is the use of qualitative research methods to explicate the complexities of participants' experiences and the meanings they hold for them. It is important to note that in this study we were not attempting to ascertain the effectiveness of the intervention or evaluate the implementation of the intervention per se, but rather to analyse the impact of a greater focus on gratitude and its perceived benefits and challenges in the context of elite sport.

\section{Research rigor}

In keeping with the constructivist-interpretivist approach we have ensured rigor by aligning our methods and data analysis with the underlying ontological and epistemological assumptions of this approach (O’Hara et al. 2011). By considering the criteria for conducting rigorous qualitative research offered by Smith and McGannon (2018), we have ensured that we are clear about not presenting a definitive picture of the social reality, but rather an interpretation of what the participants state about this reality. Acknowledging the ontological relativism in the method, we have sought multiple individual perspectives in relation to the phenomenon of gratitude. The interview data presented, using the participant's voice, showcases their experiences rather than only presenting the interpretation of the researchers. 
Our epistemological assumptions are based on a subjectivist paradigm that relies on the individual's perspective and stresses the role and contribution of the researchers' interpretation (Bradshaw, Atkinson and Doody 2017). Our use of convenience sampling and collecting data via interview facilitated emergence of interpretations of the phenomenon of gratitude. We are therefore not seeking an objective reality, but one that is reflected by the viewpoint of the participants and the researcher's interpretation of their experience. Further alignment was ensured by comparing and contrasting our results with other studies of the phenomenon in different contexts, as well as the exploration outlined in the literature.

Ethical approval for the research was approved by the Tasmanian Social Sciences Human Research Ethics Committee (Reference No. HH0009868). All the participants provided signed, informed consent to be involved in the study.

\section{Gratitude intervention}

All participants attended a full-day gratitude workshop with the following intended outcomes:

- To explore the concept of gratitude both as a state and an expression, and its meanings and challenges in the context of elite sport;

- To investigate the relevance of empirical findings on gratitude in relationship to wellbeing and relationships;

- To discuss challenges of practising gratitude in the midst of adversity and resentment; and

- To identify practices of gratitude that can be applied after the workshop. 
During the workshop, the athletes were invited to identify how they could best express gratitude to people who had supported them in their recent Rio Olympics or World Championship campaigns. To move past a tokenistic or surface expression of gratitude, participants were invited to explore ways of expressing gratitude that are authentic to them and meaningful to the person to whom they are expressing gratitude. The intervention also included the participants consciously practising gratitude in the following six weeks and then be prepared to discuss outcomes in a follow-up interview.

\section{Participants}

The participants were ten elite athletes (three male and seven female) from the sports of high-jumping, beach volleyball, diving, cycling, sailing and triathlon. Two of the athletes had competed in the Rio Paralympic Games, six had competed in the Rio Olympic Games, and two had competed in the World Championships for Cycling. Three were Olympians who had participated in two or more Games and the others had competed at the international level. There was a mix of six athletes who competed in a team sport and four who competed in individual events. Ages ranged from 20 to 42 years, with a mean age of 22 years. Participants were a sample of convenience (Miles and Huberman, 1994) because they were nominated to participate in the intervention by sporting officials and coaches. However, participation in both the workshop and the research aspect of the programme was voluntary. None of the participants had been trained specifically in the application of gratitude as defined and outlined in the intervention. All workshop participants agreed to be involved in the research.

\section{Data collection: Semi-structured interviews}

Data collection comprised qualitative interviews, which were designed to enable deep exploration of the participants' conceptions and expressions of gratitude. All ten 
participants were interviewed by telephone six weeks after the gratitude workshop. This period of time was necessary to give the participants the opportunity to reflect on and implement their gratitude practices, which have been shown to need consistent attention over a number of weeks to be effective (Howells 2012). A conversational strategy was adopted during the interviews in order to promote free and flowing conversations (Fontana and Frey 2003); however, a semi-structured interview protocol was used to guide the conversation so that the main topics to be explored were addressed by all of the participants. According to Longhurst (2003), semi-structured interviews are well suited for the exploration of the perceptions and opinions of respondents regarding complex and sometimes sensitive issues and enable probing for more information and clarification of answers. In this way rich, descriptive data are collected. Silverman (2003) warns, however, that although qualitative data collection methods, such as interview, provide valuable information about 'how people see things,' the methods often ignore the importance of 'how people do things' (359). To address this concern, the interview protocol was designed to focus on drawing out retrospective and real-time accounts (Gioia, Corley, and Hamilton 2012) of gratitude expressing and receiving from the participants.

Based on Howells' (2012) framework that embraces notions of both felt gratitude and expressed gratitude, the interview schedule asked for specific examples of how the athletes expressed gratitude. To determine the emotional impact of expressing gratitude, the interview asked, 'How does that make you feel when you express gratitude?’ To further interrogate the relational dimensions of gratitude (Howells 2012), participants were also asked, 'What impact do you think gratitude is having on your relationships?' Then they were asked for specific examples in their sport. To capture the symbiotic relationship between giving and receiving gratitude (Howells and Cumming 
2012), the athletes were asked, 'Have you noticed more gratitude expressed to you?' They were also asked, 'Have you noticed any impact on your performance from practising gratitude?' Similar questions were asked about the perceived impact on stress levels. As the literature explores challenges around forced obligation and expected reciprocity (Buck 2004, Smilansky 2002), the interview also interrogated these challenges, particularly when the athletes expressed gratitude. Throughout the interview the participants were prompted further by being asked to 'Tell me more about that' or 'Can you give me an example?’ as is appropriate for semi-structured interviews (Fontana and Frey 2003, Longhurst 2003). The aim was to collect data that would enable greater understanding of the important contextual influences on how gratitude is expressed and the challenges of doing so.

Although telephone interviews can be impersonal, the interviewer, who was the lead researcher, was familiar with the athletes as she had also been the facilitator of the workshop. Because responding to the questions pertaining to gratitude required a trusting relationship, it was decided that prior-knowledge of the interviewer was important to allow for open and free dialogue. To maintain impartiality, it was made clear at the beginning of the interviews that they were being conducted in the spirit of research and the intention was to represent their experiences faithfully. Two of the participants requested to be interviewed together and permission was given for this on the proviso that they gave individual answers and tried not to be influenced by the opinions of the other. The average duration of the interviews was 40 minutes.

All interviews were audio-recorded and professionally transcribed verbatim. Once transcribed, interviews were sent to the participants to check for accuracy and were invited to add to or change the documents. Two of the participants requested minor changes be made. These were mostly typographical due to misinterpretation of 
spoken words by the transcriber. This member-checking strategy contributed to the robustness of the research (Smith and McGannon 2018).

\section{Data Analysis}

Content analysis was used to probe the semi-structured interviews. Content analysis comprises three distinct data analysis approaches—conventional, directed, or summative — and is a widely used qualitative research technique (Hsieh and Shannon 2005). The conventional content analysis involved line by line open coding using an inductive approach (Strauss and Corbin 1998) to identify interesting aspects in the data as in participants' terms (Braun and Clarke 2006). As recommended by Gioia, Corley, and Hamilton (2012), at this stage, little attempt was made to distil the data into emerging patterns and categories. The data, however, were collated according to the codes applied (Braun and Clarke 2006).

Directed content analysis was then conducted in accordance with the data analysis process detailed by Hsieh and Shannon (2015), which is similar to what Strauss and Corbin (1998) describe as axial coding. This process was used to identify the patterns and categories that arose from the first round of coding. This 2nd-order analysis was distilled further to determine the main themes from the data (Braun and Clarke 2006), which contribute to the theorising presented in the Discussion (Gioia, Corley, and Hamilton 2012). This process is in keeping with the interpretivist/ constructivist because it honours the participants' voice and keeps the results grounded in the data. Summative content analysis (Hsieh and Shannon 2005) was not conducted due to its incompatibility with the relatively small sample size. To protect the privacy of the participants, pseudonyms were used to report the participants' contributions to the research. 


\section{Findings}

Analysis of the interview data revealed several interrelated themes regarding outcomes of the intervention, which included attending the workshop and a six-week period of practising gratitude, and the perceived challenges in practising gratitude in the context of elite sport. Our two broad categories for reporting the data are the impact of practising gratitude and the challenges in practising gratitude. Each of the themes and related sub-themes that fall under these categories are listed in Table 1, described below and illustrated with indicative statements from participants.

Table 1. Data codes

\begin{tabular}{|c|c|c|}
\hline Category & Theme & Sub-themes \\
\hline \multirow[t]{4}{*}{$\begin{array}{l}\text { Impact of practicing } \\
\text { gratitude }\end{array}$} & Improved attitude & $\begin{array}{l}\text { Choosing their state of being } \\
\text { Shifting from negative to positive } \\
\text { Reflection before training } \\
\text { Improving their relationships with others }\end{array}$ \\
\hline & Greater awareness & $\begin{array}{l}\text { Awareness of the contribution of others, and } \\
\text { Increased awareness of what had been taken for } \\
\text { granted in the past }\end{array}$ \\
\hline & Better capacity to deal with stre & $\begin{array}{l}\text { Improved resilience moment } \\
\text { Increased calmness } \\
\text { Greater ability to be in the present moment }\end{array}$ \\
\hline & Improved performance & $\begin{array}{l}\text { Resilience } \\
\text { Improved mental state } \\
\text { Teamwork }\end{array}$ \\
\hline \multirow[t]{3}{*}{$\begin{array}{l}\text { Challenges in } \\
\text { practising gratitude }\end{array}$} & Influence of context & $\begin{array}{l}\text { Single-minded nature of competitive sport } \\
\text { Individualistic nature of some sporting contexts }\end{array}$ \\
\hline & $\begin{array}{l}\text { Expressing gratitude in } \\
\text { meaningful and appropriate ways }\end{array}$ & $\begin{array}{l}\text { Awkwardness in giving and receiving gratitude } \\
\text { Lack of opportunities }\end{array}$ \\
\hline & $\begin{array}{l}\text { Lack of perceived value from } \\
\text { others }\end{array}$ & $\begin{array}{l}\text { Heightened awareness of lack of gratitude from } \\
\text { others } \\
\text { Managing one's negativity when they or others } \\
\text { did not receive gratitude }\end{array}$ \\
\hline
\end{tabular}

\section{Impact of practising gratitude}

\section{Improved attitude}

Most of the athletes said that their increased attention to gratitude had a positive impact on their attitude, which in turn, assisted them with the performance, particularly in the preparation stage. Here the term 'attitude' is defined as the kind of lens we look through to orientate ourselves in the world. Other terms commonly used synonymously are 
'inner outlook' or perspective. The athletes often used the term 'mindset'. This change in attitude was discussed in terms of decision-making; life-choices; reassessing life; 'being able to take stock'; and using reflection more consciously.

The most valued shift in attitude was attributed to strategies of moving from resentment towards gratitude. Most found the shift in their attitude from one of resentment, to be the most effective aspect of a greater attention to gratitude. This aligns with the conceptual work of Roberts (2004), which demonstrates the symmetrical relationship between the two states, and was an aspect of gratitude that was explored in the workshop. An important emphasis was that of gratitude not being seen as a way of eliminating resentment, but rather as a means of identifying where gratitude may be difficult and therefore act more proactively on this.

Ways in which the athletes reported addressing their resentment proactively included being able to put oneself in another's shoes; taking a step back from the situation and reflecting; not joining in with gossip or backbiting; speaking more directly about grievances with the aim of resolving them; having conversations of mutual respect; and giving priority to strengthening relationships.

The sub-themes that arose from the analysis were: choosing their state of being; shifting from negative to positive; reflection before training; and improving their relationships with others. Gratitude gave the athletes a greater sense of empowerment to choose their state of being, which in turn influenced their reactions, or as Gabrielle put it, her ability to 'use it as an assessment of how you view things and everything in your life.' When interrogated a little further about how this would apply to her sporting context, she said that increased gratitude 'helps you to pause and reassess and see what you can do better' and therefore 'go about with a better outlook.' This sub-theme is exemplified in Nathan's estimation of what he would do differently in the future, where: 
If I don't have a performance that I'm happy with, it's being able to respond to everybody in a different sort of way to what one of my training partners did, in that you want to acknowledge that people still have watched and supported me.

Some discussed the change in attitude as a shift from negative to positive. Tim found value in gratitude helping him to go '...into a race and not thinking about negatives, not focusing on whether you're feeling sore or whether you've been struggling.' He used gratitude to put his 'mind in a better place' and taking it out of a 'bad place.' Although she had not yet experienced such a shift, Millie saw the greatest potential for gratitude in the domain of changing one's attitude, 'particularly when you are in a bad mood.' A more positive attitude afforded by increased gratitude was discussed by Ruby, as having a 'fresh start' in each new training session, 'whether it's been good or bad in the previous session.’

Another sub-theme was how the athletes used this change in perspective to reflect on their attitude before a competition or during training. For example, when she was in the care on the way to training, Ruby reflected on how she could bring a grateful perspective to her training, 'How do I make sure my coach feels like I am grateful for him coming to the training session?'

Most of the athletes highlighted the benefits of this shift for their relationships with others - including team mates and coaches. Sue discussed the value of this change in attitude in terms of adding clarity and said, 'Why I feel a certain way towards someone and why I remove myself from them or I take a step back from them.’ Ruby felt that increased gratitude assisted her to deal with disagreements at the end of training and so that she no longer dwells on negative things that may have happened in communications with her coach. Tim's attempts to refrain from backbiting or gossiping 
and not listening to other's doing this led to a direct impact on relationships and therefore performance because he was less distracted.

Not getting caught up in stupid, stuff that other people are doing, which all that does is distract me from what I need to do to get the best out of me. I feel it's just helping me stay focused.... I'm not distracted by other things that are going on, I'm just focusing on myself and what I need to do.

\section{Greater awareness}

The changed perspective was also discussed in terms of being more self-aware, as well as greater awareness of the things to be grateful for. The sub-themes are greater awareness of the contribution of others, and increased awareness of what had been taken for granted in the past.

Regarding the first sub-theme of greater awareness of the contribution of others, particular mention was made about the consequent increased awareness of their interconnectedness with others and the opportunities that they had been given. For example, Ruby felt 'more aware of not only myself, but everyone else around me.' Belinda, who had been struggling with having to move back home with her parents, was able to shift her awareness to what she was given in this situation, where, 'I realised that I get to spend time with my mum and dad, and I have a roof over my head and I'm really happy here.' This aligns with the extensive body of literature which highlights the capacity for gratitude to build and maintain relationships, while at the same time growing an awareness of what one has received from others (Algoe, 2012).

Increased attention to gratitude also assisted in greater awareness of things that the athletes had taken for granted in the past. Ruby said that the biggest outcome for her was that 'I'm just more aware...I feel in myself that I'm more grateful for little things, not just when you kind of think you should be grateful for something big, but just like 
the little things I now notice.' Ruby also discussed this from the view of the sporting context when she recounted speaking up at a training camp. For her, it was

...about making sure we all understand that doing our sport is a privilege, and being grateful for the opportunity to travel interstate, or to compete overseas, or even just being able to come to training and have a coach coaching you every day.

\section{Better capacity to deal with stress}

For many of the athletes, a direct outcome of increased attention to gratitude was the improved ability to deal with stress. The three sub-themes were the impact on improved resilience; increased calmness; and greater ability to be in the present moment. Such findings align with other studies that explore the positive impact of gratitude on stress in other contexts (for example, Vieselmeyer, Holguin, and Mezulis 2016, Wood, Joseph, and Maltby 2009).

Regarding the impact on resilience, as a result of changing her attitude to one of gratitude, Ruby said she had a 'clearer head and lighter.' This is in contrast to the past where, '...I've brought bad emotions into training that have nothing to do with training but have just carried with me throughout the day, it makes me feel tired, it makes me feel heavy.' She felt that when she changed her attitude to gratitude 'if I come to any little curve balls in training, I feel more capable to get through them because I'm already in a better mindset.'

Gabrielle reported that I think that definitely comes into play when you tap into that gratitude and you're like, Wait a minute, it's all right. Just keep on persisting and remember what you are blessed with...And that helps you to get through hard times. It helps, having that faith in yourself and being able to meet what comes when...In terms of your stress levels on the whole. 
Her response indicates several areas that she deems relevant to the use of gratitude to increase resilience: being able to stand back; having faith in yourself; and "being able to meet what comes.”

A specific outcome reported as a result in a shift in attitude was that many of the athletes felt calmer, which in turn, had an impact on stress levels. This is a common theme in other qualitative research on the impact of gratitude (Howells 2014, 2017; Howells and Cumming, 2012). What this data provides us with is more detail about the link between gratitude and calmness, and how it manifests within this context. In particular, many of the athletes linked an increased sense of calmness to how their increased gratitude led to better relationships with others. For Sue, a 'better feeling of calm and control' arose because 'it helps steady the ship almost... There's a sense of calmness rather than anxiety, or uncertainty, or animosity.' For others, the calmness arose from a change in attitude relating to their personal stress. For example, gratitude assisted Belinda to 'be open-minded and really think about what you really have helps you calm down and be proactive.' For Emma 'gratitude thoughts' are 'calming because they give me a sense that we are capable, I am capable, we do have a very good opportunity to go and achieve what we want to achieve.'

Both Sue and Gabrielle, discussed a reduction in stress arising from the sense that gratitude assists one to be more fully in the present where, as Gabrielle says, 'You feel gratitude in your heart and your love in the moment.' This in turn had an impact on her performance:

I think that's key when you're performing because you're in the moment, you're not stressing about the past, you're not stressing about the future, you're just enjoying each and every blessing you have.... So, gratitude gets you in the right state of mind to be able to perform at your best, I believe. 
Greater attention to gratitude assisted Sue to be more present because she felt more connected to others. She reported that gratitude assists greatly with being 'are present and are mindful of what people around you are doing,' which in turn assists her to be more 'balanced and grounded.'

\section{Increased confidence to express gratitude}

Being able to address resentment in proactive ways as a means of increasing gratitude was one significant way in which nearly all the athletes grew in confidence, and this enabled them to improve their relationships. This in turn gave them greater confidence in the expression of gratitude; assisted with their overall communication skills; and improved their relationships - the three sub-themes under this category.

The athletes found that as a result of the program they became both more confident and more aware of expressing more gratitude to others, as they discussed thanking individuals, such as their coaches, partners, parents, grandparents, people in sporting organisations, fans, and others who support them. They discussed different means of expressing their gratitude, such as offering more sincere verbal thanks, writing letters to their coach, sending flowers, or taking their mother out for breakfast, or caring more for their partners and parents. Sue gave a moving example of this:

This morning, a lady at the gym helped me get my bike in, which was not normal practise, because you need to get your bike out. Its little every day gestures when you are more in the moment, and you're observing this person doing this, and you have a nice conversation, and you're able to say, 'Thank you so much.' Then you've just built another relationship with another stranger. All of that helps you in your environment, and your training; when you're more aware of your daily interaction and what people are doing to help you. 
Another case in point was Nathan's account of thanking his physio and massage therapist whom he had worked with for years, but never thanked. Nathan expressed gratitude, 'In specific ways' that he had not thought of doing in the past, 'and actually spending time thanking them for what they’ve done.' In response the massage therapist said that:

...in his 20 years of doing massage therapy, he said very rarely you get athletes coming in and actually saying thank you to him, which I did, and he was really happy with that. He said it was not a very normal thing that they get, so I think that was one thing that I thought of.

As the athletes became better at expressing gratitude, this had a positive impact on both their confidence and communication skills more generally. For example, Gabrielle thanked her coach through a letter because:

He's a pretty quiet man, but he was appreciative of my note. He always enjoys the written word, and I'm a quiet person, too, so sometimes I do find it hard to express my feelings, so by doing it that way it was helping me as well as helping him.

This in turn helped her communication skills.

Expressing my feelings, I'm not very good at, and so I think that definitely helped me. Because I guess I was looking at how he wanted it done. Not wanted it done but liked to be shown appreciation. But it also helped me to see my own thoughts and really express and write what I felt and be able to deliver my gratitude in that aspect.

Tim became better at communicating with his coach:

I've been a lot better with my coach and communicating with him better and showing gratitude towards him for just how he's helped me. Not even just him, with other people like my family and things like that have improved in that sort of sense and been more aware of it, I guess. 
The improved communication skills were discussed in terms of clarity of to whom the athletes were to express gratitude, and greater sincerity. They were better able to express gratitude to those whom they had realised should receive their thanks and take advantage of situations where this is called for, including towards organisations such as schools and sporting bodies. Participants also discussed being able to express gratitude in more meaningful and sincere ways. Both the quantity and the quality of expressed gratitude was reported to have increased. As Ruby said:

I say thank you quite a bit more now ...I feel like I can reciprocate doing small things to help other people, and that shows, I think, even more than saying thank you, that you're grateful for anything that people do.

Some athletes increased their sincerity in expressing gratitude. Lily reported shifting to expressing gratitude 'Not just for the sake of it, but because I actually mean it as well.'

As a consequence of the increased confidence in expressing gratitude the athletes found that they improved their relationships. As we see from the above examples of Sue and Nathan, we can see the capacity of gratitude to build and maintain relationships as outlined in Algoe’s (2012) theory. For Sue, increased gratitude grew her awareness of the other and deepened that relationship. For Nathan there was an extension of this awareness through receiving thanks from the person to whom he was expressing gratitude.

\section{Improved performance}

Although some of the participants stated that it was difficult for them to ascertain the impact of the workshop on their performance because it was the time of the year when things were winding down, those who were still having opportunities to perform stated 
that they noted positive effects. Areas that were mentioned include a positive impact on resilience, improved mental state; and teamwork- the three sub-themes under this category. As we have seen from the above analysis, factors such as a change in attitude, a greater capacity to deal with stress, and an increased attention to the present moment, have all been reported to have a positive impact on performance. Here we investigate other aspects that have been emphasised by the participants as they relate to the subthemes.

Some participants attributed their improved performance to their increased ability to address their resentment. This in turn impacted on resilience. For example, Ruby said that:

I think it definitely has a positive impact on my performance, because I come back to training the next day, whether it's been a good or a bad previous session, on a fresh start and still working towards new goals and with that clear mindset...compared to if I came in still upset with my coach from the day before because we hadn't ended on a good note...it's definitely improved my mental performance.

And as Tim stated, Yeah, it's well and truly an impact from giving up resentment because you just ... if you're worried about that and using that resentment and stuff it's just wasted energy almost whereas without that, it's just something I'm not even worrying about. I can focus that straight back into my performance and not be wasting energy worrying about silly things that are going on around me.

The impact of gratitude on their mental state and how this consequently impacted on their performance or training was a focus for the participants. Gabrielle said that gratitude helped her feel 'more alive' by experiencing more gratitude in the moments she was training. Tim stated that 'I definitely feel like it helps me improve my 
racing for the week because I was just in a really good mindset for the entire week after that.' For Belinda,

Absolutely, absolutely. I think, for me, that real central thing is that idea that we spend so much time and energy on what we don't like, on what doesn't align with our perfect world...I think that that's where understanding the place of gratitude and practising that really gives us that boost, that mental and emotional boost that's critical to high performance psychology and positive health and well-being.

Tim noted that increased attention to gratitude assisted his ability to work better with his fellow team mate, where,

I guess because we race together a lot as team mates, we've just become closer as team mates which in turn has helped us on the track racing because it's just increased the trust between the two of us which is something that is important between team mates. I feel, in that respect it would have helped our performance, just we've got more trust in each other now.

\section{Challenges in practising gratitude}

Three interrelated themes were found under this category: the influences of context; inability to express gratitude in meaningful and appropriate ways; and lack of perceived value from others.

The influence of context

Regarding the influence of context, some of these athletes commented upon the nature of competitive sport not necessarily being conducive to expressing gratitude. There are two interrelated sub-themes in the way this was challenge was discussed: the singleminded nature of competitive sport and the individualistic nature of some sporting contexts. 
An aspect of the single-minded nature of elite sport that was considered to be prohibitive was the pressure to be focused on the future rather than the present moment. As Gabrielle stated,

I guess sometimes the gratitude is harder to see when you're putting too much emphasis and stress on the future and trying to make decisions count too much instead of just living in the moment and listening to your heart and being grateful for everything.

Sue put this factor down to the busy environment and one where 'you're already thinking about where you've got to go next, and you're not staying in the moment and taking the time in to allow people to express their gratitude, and you to receive it.

In regard to the sub-theme of the individualistic nature of some elite sporting contexts, the focus and mindset needed to succeed were spoken about as being quite individualistic and seemed to preclude the prosocial nature of a practice such as gratitude, which focuses on the other. Further aspects that were seen to make gratitude difficult in terms of the context were the lack of opportunities to focus on relationships because of the singlemindedness required to become an elite athlete; the competitive spirit required; and the stresses and distraction that come with such a focus. Variables in these aspects were mentioned in terms of whether competing in a team sport or an individual sport. For example, Tim said that 'it seems difficult to focus on both the event and one's gratitude to others at the same time.' However, he mentioned he has made it one of his aims.

Expressing gratitude in appropriate and meaningful ways Another theme under challenges relates to expressing gratitude in appropriate and meaningful ways. The athletes reported that they felt awkward when trying to find appropriate and sincere ways of expressing gratitude. Some participants reported the 
need to focus on the relationship first before being able to express gratitude with confidence, and again given the nature of the single-mindedness needed in elite sport, they found this to be challenging. One particular aspect was the notion that expressing gratitude could be mis-conceived by recipients as trying to 'suck up.' This was discussed in respect to the athlete-coach relationship. Some of the athletes reported that it was awkward to express gratitude when others did not know how to receive it or that they felt uncomfortable with giving or receiving gratitude, or when it did not appear to mean anything to others. They also noted that some people had that same awkwardness in expressing gratitude towards them. As Peter reported that it seemed like an insult when a coach did not receive his gratitude graciously.

But I think maybe the main challenge for me which will probably be for many people across the board is just the uncomfortableness of it a lot of the time. If a person is uncomfortable with giving or receiving gratitude, it's a little bit awkward when you're trying to express gratitude. I think all round just the uncomfortable aspect of opening yourself up a little bit in a more personal way, which is what you feel like you are doing when you are expressing sincere gratitude.

The other sub-theme was how they found it difficult at times to find the opportunity to express gratitude. For example, Millie discussed the nature of sporting events themselves, where it is often difficult to return gratitude to the individuals who contributed to the success; volunteers who worked at the Rio Olympics was one case in point.

The lack of perceived value from others

The last theme, lack of perceived value from others, was discussed in terms of a heightened awareness of when they did not receive gratitude from others; and difficulty 
with self-management when they, or other athletes, did not receive gratitude from others. Some reported that there was greater awareness of others not expressing gratitude to them and thus the program heightened their sensitivity to this and highlighted areas where they would have expected gratitude but did not receive it. Some athletes reported the challenge of managing their negativity or resentment when they or other athletes did not receive gratitude from others. As Belinda noted, 'Right now, postOlympics, to see athletes feel undervalued and not sure that they want to keep going. Because they feel this, terrifies me. That's where I think gratitude needs to play a bigger role in sports.'

\section{Discussion and Conclusion}

The purpose of this study was to investigate the impact of an intervention designed to encourage a greater focus on gratitude in elite athletes. Results of the study showed that gratitude — conceptualised as an active practice of giving back and as the opposite of resentment—was seen by the athletes as highly relevant in the contexts of their professional and personal lives. The program provided the opportunity for the participants to discover various dimensions of gratitude that enhance their personal and relational wellbeing. Consequently, for some this meant improved performance. The aspects of performance that were reported to improve were a change in perspective, dealing with stress, improved team-work, and better communication in the athlete-coach relationship. These outcomes indicate that increased attention to gratitude can have a direct influence on factors that are highly relevant to the sporting world. This may be secondary, however, to the enhanced wellbeing and increased skill in communicating and relating with others reported in this study and that are gained just for their own sake. Outcomes may also be relevant to elite athletes as they transition out of the sport, where a greater emphasis on support is needed (Beable et al. 2017). 
The positive outcomes reported by the athletes in this study relate to the wider literature on the benefits of gratitude and thus broaden our understanding of its potential relevance for elite athletes. In particular, reported improved emotional and mental wellbeing offers further validation to studies that report on positive effects of gratitude for health and wellbeing (e.g., McCullough, Emmons and Tsang 2002, Watkins, Uhder, and Pichinevskiy 2015). Further, the literature on the prosocial benefits of gratitude (e.g., Algoe 2012, Tsang and Martin 2019, Yost-Dubrow and Dunham 2018), accounts for the findings in this study where athletes reported greater relational wellbeing.

The study also adds a qualitative lens to research on gratitude in sport, which to date has relied on quantitative methods. Although the existing research points to the complex nature of gratitude and how it may be shaped by a range of factors (Lambert, Graham and Fincham, 2009; McConnell 2016), the present study provides insight into how this complexity is experienced by elite athletes. These include particular contextual influences such as the single-mindedness and focus that are required and which may preclude a focus on expressing gratitude in relationships or attending to anything other than performance.

Insights offered into the complexities of expressing gratitude have particular relevance to the coach-athlete relationship and the power imbalance that is often characteristic of this. Because of the high-stakes relationship between athlete and coach, how does the elite athlete express gratitude authentically when there are undertones of power dynamics at play and the looming threat of possible non-selection for major sporting events? How do athletes express gratitude when the coach is not good at receiving it, and what are the consequent outcomes for communication and relationships? 
The positive outcomes reported in this study also interrelate with those reported in other educational environments. For instance, where research students report greater awareness and improved cognitive ability (Froh et al. 2009; Howells et al. 2017), these athletes report the same but with an emphasis on performance and mindset. Where teacher-student relationships are shown to have greatly improved through the application of gratitude, and especially through attending proactively to resentment (Howells 2017, 2018; Howells et al. 2017), these athletes have reported that their practice of gratitude also greatly improved their relationships and communication. Thus, the present study affirms that greater attention to expressed gratitude provides confidence and skill in building and maintaining relationships.

These findings have relevance for efforts to improve the health and wellbeing of elite athletes. A growing body of research supports the view that social connections play a key part in helping elite athletes to achieve high levels of performance in ways that do not jeopardise their long-term health (Fletcher and Streeter 2016, Grahn 2014). Thus, it is important to understand how the relational benefits of gratitude may best be harnessed to support these goals. In addition, the positive outcomes reported here have significance for current policy directives to foster more holistic approaches to coaching and athlete development. For example, a recent International Olympic Committee consensus statement on youth athletic development (Bergeron et al. 2015) noted that, in addition to the ability to teach sport-specific skills, 'coaching effectiveness is also reflected in the ability to create and maintain relationships with others' (7). The Committee also recommended that 'coaching education programmes should assist coaches in establishing meaningful relationships that enrich the personal assets of their athletes and foster their own intrapersonal and interpersonal skills' (8). The benefits of expressing gratitude as reported by athletes in the present study suggest that such 
practices may also be of value in re-orienting coaching practices within elite sport to encompass these relational skills.

Further significance of the study lies in its description of an actual intervention in which athletes were invited to pay greater attention to expressed gratitude in sporting and everyday contexts. As discussed previously, existing research in this area has focussed on the role of dispositional or felt gratitude. However, as Yoshimura and Berzins $(2017,110)$ note, gratitude is primarily a social emotion and therefore 'beneficial not only because it is experienced but because it is actually expressed.' While previous studies have investigated the impact of interventions to facilitate the expression of gratitude in a range of contexts (Dickens 2017), this is the first study to do so in the context of elite sport. As such, the study provides important insight into the benefits and challenges of gratitude interventions for elite athletes. Further, the study aligns with previous research in which it is argued that the way in which gratitude is framed and researched must acknowledge the complexity of the construct (Lambert, Graham, and Fincham 2009, Manela 2016), and in doing so must take proper account of the underlying difficulties in practising gratitude within particular contexts (Howells 2012, 2014b, 2015).

Accordingly, a key concern in the present study was to explore the challenges participants encountered in practising gratitude in the elite sporting context. Among the challenges reported by these athletes were concerns about how gratitude can be expressed in environments that are traditionally focussed on competition and the end point of winning. These concerns resonate with recent studies of elite sporting culture, which have drawn attention to the negative impact that such a focus on performance outcomes can have for the health and wellbeing of elite athletes (e.g., Barker et al. 2014, Carless and Douglas 2012). In these studies, narrow conceptualisations of sporting 
success in terms of performance outcomes are seen to undermine the importance of healthy relationships and strong social connections within elite sport. According to Carless and Douglas (2012), the dominance of the performance narrative within elite sporting culture functions to marginalise or silence alternative conceptions of sporting success, such as those contained within the relational narrative, where 'being with other/s is storied as more important than achievement in terms of wins or trophies' (394).

The gratitude program was talked about positively by all ten athletes and there were many comments about its relevance to coaches and to the system. When practising gratitude because of what was learned in the workshop, the athletes reported benefits for their own personal outlook, as well as confidence and skills in being able to express gratitude towards others. In sum, the findings from the present study demonstrate that gratitude-based interventions can play an important part in supporting the health and wellbeing of elite athletes.

\section{Limitations and Future Directions}

As this was a small study, it is not possible to extrapolate the findings to larger populations (Gioia, Corley, and Hamilton 2012, Smith, 2018). However, the in-depth qualitative investigation of just a few athletes has generated results that suggest it would be worthwhile to explore further the role of greater attention to gratitude in elite sport. As the literature reports widely on the concern for stress and burnout of athletes (Gustafsson, Sagar, and Stenling 2017), the reported positive outcomes in this study in the domain of addressing stress warrants further attention in future research. Also, as gratitude was reported by these athletes to have had a significant impact on their relationships and communication, a specific focus on the role of gratitude in the athletecoach relationship would also be beneficial. 


\section{References}

Algoe, S. B. 2012. "Find, Remind, and Bind: The Functions of Gratitude in Everyday Relationships." Social and Personality Psychology Compass 6 (6): 455-469. doi:10.1111/j.1751-9004.2012.00439.x

Algoe, S. B., J. Haidt, and S. Gable. 2008. "Beyond Reciprocity: Gratitude and Relationships in Everyday Life." Emotion 8 (3): 425-429.

Alkozei, A., R. Smith, M. D. Kotzin, D. L. Waugaman, and W. D. S. Killgore. 2017. "The Association Between Trait Gratitude and Self-Reported Sleep Quality is Mediated by Depressive Mood State." Behavioral Sleep Medicine 27: 1-9. doi:10.1080/15402002.2016.1276017

Annerstedt, C., and E-C. Lindgren. 2014. "Caring as an Important Foundation in Coaching for Social Sustainability: A Case Study of a Successful Swedish Coach in High-Performance Sport." Reflective Practice 15 (1): 27-39. doi:10.1080/14623943.2013.869204

Barker, D., N. Barker-Ruchti, A. Wals, and R. Tinning. 2014. "High Performance Sport and Sustainability: A Contradiction of Terms?" Reflective Practice 15 (1): 1-11. doi:10.1080/14623943.2013.868799

Bartlett, M. Y., and D. Desteno. 2006. "Gratitude and Prosocial Behavior: Helping When it Costs You." Psychological Science 17 (4): 319-325. doi:10.1111/j.1467-9280.2006.01705.x

Beable, S., M. Fulcher, A.C. Lee, and B. Hamilton. 2017. "SHARPSports Mental Health Awareness Research Project: Prevalence and Risk Factors of Depressive Symptoms and Life Stress in Elite Athletes.” Journal of Science and Medicine in Sport 20 (12):1047-1052. doi:10.1016/j.jsams.2017.04.018

Benson, A. J., P. Šiška, M. Eys, S. Priklerová, and P. Slepička. 2016. "A Prospective Multilevel Examination of the Relationship Between Cohesion and Team Performance in Elite Youth Sport." Psychology of Sport and Exercise 27(Supplement C): 39-46. doi:10.1016/j.psychsport.2016.07.009

Bergeron, M. F., M. Mountjoy, N. Armstrong, M. Chia. J. Côté, C. A. Emery, A. Faigenbaum, et al. 2015. "International Olympic Committee Consensus Statement on Youth Athletic Development." British Journal of Sports Medicine, 49: 843-851.

Bradshaw, C., S. Atkinson, and O. Doody. 2017. " Employing a Qualitative Description Approach in Health Care Research." Global Qualitative Nursing Research.[Pulished online first] doi:10.1177/2333393617742282

Braun, V., and V. Clarke. 2006. "Using thematic analysis in psychology." Qualitative Research in Psychology 3 (2) 77-101.

Buck, R. 2004. "The Gratitude of Exchange and the Gratitude of Caring: A DevelopmentalInteractionist Perspective of Moral Emotion." In The Psychology of Gratitude, edited by R. Emmons, and M. McCullough, 100-122. Oxford: Oxford University Press.

Busanich, R., K. R. McGannon, and R. J. Schinke. 2016. "Exploring Disordered Eating and Embodiment in Male Distance Runners Through Visual Narrative Methods." Qualitative Research in Sport, Exercise and Health 8 (1): 95-112. doi:10.1080/2159676x.2015.1028093

Carless, D., and K. Douglas. 2012. "Stories of Success: Cultural Narratives and Personal Stories of Elite and Professional Athletes." Reflective Practice, 13 (3): 387-398. doi:10.1080/14623943.2012.657793

Chen, L. H. 2013. "Gratitude and Adolescent Athletes’ Well-Being: The Multiple Mediating Roles of Perceived Social Support From Coaches and Teammates." Social Indicators Research 114 (2): 273-285. doi:10.1007/s11205-012-0145-2

Chen, L. H., and Y. K. Chang. 2014. "Cross-Lagged Associations Between Gratitude and Adolescent Burnout." Current Psychology 33: 460-478.

Chen, L. H., and Chang, Y.-P. 2017. "Sport-Domain Gratitude Uniquely Accounts for Athletes' Well-Being Across Two Cultures: Incremental Validity Above the General Gratitude." The Journal of Positive Psychology 12 (6): 651-659. doi:10.1080/17439760.2016.1257052

Chen, L. H., and Y. H. Kee. 2008. "Gratitude and Adolescent Athletes' Wellbeing." Social Indicators Research 89: 361-373. 
Chen, L. H., and C.-H. Wu. 2014. "Gratitude Enhances Change in Athletes' Self-Esteem: The Moderating Role of Trust in Coach." Journal of Applied Sport Psychology 26 (3): 349-362. doi:10.1080/10413200.2014.889255

Creswell, J. W. 2014. Research Design: Qualitative, Quantitative and Mixed Methods Approaches. 4th ed. Thousand Oaks, CA: SAGE Publications.

Dickens, L. R. 2017. "Using Gratitude to Promote Positive Change: A Series of Meta-Analyses Investigating the Effectiveness of Gratitude Interventions." Basic and Applied Social Psychology 39 (4): 193-208. doi:10.1080/01973533.2017.1323638

Doherty, S., B. Hannigan, and M. J. Campbell. 2016. "The Experience of Depression During the Careers of Elite Male Athletes." Frontiers in Psychology 7: 1069. doi:10.3389/fpsyg.2016.01069

Douglas, K., and D. Carless. 2009. "Abandoning the Performance Narrative: Two Women's Stories of Transition From Professional Sport." Journal of Applied Sport Psychology 21 (2): 213-230. doi:10.1080/10413200902795109

Emmons, R. A., and M. E. McCullough. 2003. "Counting Blessings Versus Burdens: An Experimental Investigation of Gratitude and Subjective Well-Being in Daily Life." Journal of Personality and Social Psychology 84 (2): 377-389.

Filho, E., U. Dobersek, L. Gershgoren, B. Becker, and G. Tenenbaum. 2014. "The CohesionPerformance Relationship in Sport: A 10-Year Retrospective Meta-Analysis." Sport Sciences for Health 10 (3): 165-177. doi:10.1007/s11332-014-0188-7

Fletcher, D., and A. Streeter. 2016. "A Case Study Analysis of a High Performance Environment in Elite Swimming." Journal of Change Management 16 (2): 123-141. doi:10.1080/14697017.2015.1128470

Fontana, A., and J. Frey. 2003. "The Interview: From Structured Questions to Negotiated Text." In Collecting and interpreting qualitative materials, edited by N. K. Denz,in and Y. S. Lincoln, 61-106. Thousand Oaks, CA: SAGE Publications.

Froh, J. J., T. B. Kashdan, K. M. Ozimkowski, and N. Miller. 2009. "Who Benefits the Most from a Gratitude Intervention in Children and Adolescents? Examining Positive Affect as a Moderator." The Journal of Positive Psychology, 4 (5): 408-422.

Gabana, N. T., J. A.Steinfeldt, Y. J. Wong, and Y. B. Chung. 2017. "Gratitude, Burnout, and Sport Satisfaction Among College Student-Athletes: The Mediating Role of Perceived Social Support." Journal of Clinical Sport Psychology 11(1): 14-33. doi:10.1123/jcsp.2016-0011

Galli, N., and J. J. Reel. 2012. "'It was Hard, But it was Good': A Qualitative Exploration of Stress-Related Growth in Division I Intercollegiate Athletes." Qualitative Research in Sport, Exercise and Health, 4 (3): 297-319. doi:10.1080/2159676x.2012.693524

Giel, K. E., A. Hermann-Werner, J. Mayer, K. Diehl, S. Schneider, A. Thiel, and S. Zipfel. 2016. "Eating Disorder Pathology in Elite Adolescent Athletes." International Journal of Eating Disorders 49 (6): 553-562. doi:10.1002/eat.22511

Gioia, D. A., K. G. Corley, and A. L. Hamilton. 2012. "Seeking qualitative rigor in inductive research: Notes on the Gioia methodology." Organizational Research Methods 16 (1): 1531.

Grahn, K. 2014. "Alternative Discourses in the Coaching of High Performance Youth Sport: Exploring Language of Sustainability." Reflective Practice 15 (1): 40-52. doi:10.1080/14623943.2013.868795

Gustafsson, H., S. S. Sagar, and A. Stenling. 2017. "Fear of Failure, Psychological Stress, and Burnout Among Adolescent Athletes Competing in High Level Sport." Scandinavian Journal of Medicine \& Science in Sports 27 (12): 2091-2102. doi:10.1111/sms.12797

Hodge, K., H. Graham, and W. Smith. 2014. "A Case Study of Excellence in Elite Sport: Motivational Climate in a World Champion Team." The Sport Psychologist, 28 (1): 60-74. doi:10.1123/tsp.2013-0037

Hodge, K., C. Lonsdale, and J. Y. Y. Ng. 2008. "Burnout in Elite Rugby: Relationships With Basic Psychological Needs Fulfilment." Journal of Sports Sciences 26 (8): 835-844. doi:10.1080/02640410701784525

Howells, K. 2012. Gratitude in Education: A Radical View. Rotterdam, Netherlands: Sense Publishers. 
Howells, K. 2014a. "An Exploration of the Role of Gratitude in Enhancing Teacher-Student Relationships." Teaching and Teacher Education 42: 58-67.

Howells, K. 2014b. Exploring cultural diversity through the lens of the practice of gratitude in education. The International Journal of Diversity in Education 13: 41-52.

Howells, K. M. 2015. "Researching the Place of Gratitude in the Personal Domain of the Educator: Tales from the Field." In Mainstreams, Margins and the Spaces In-between: New possibilities for Education Research, edited by K. Trimmer, A. Black, and S. Riddle, 36-49. London: Routledge.

Howells, K. 2017. "The Use of Dialogical Inquiry to Acknowledge Diversity When Researching Gratitude in Indigenous Cultures." SAGE Research Methods Cases Part 2. doi:10.4135/9781473997851

Howells, K. 2018. "Developing gratitude as a practice for teachers." In Developing gratitude in children and adolescents, edited by J. Tudge, and L. Freitas, 240-261. Cambridge: Cambridge University Press.

Howells, K., and J. Cumming. 2012. "Exploring the Role of Gratitude in the Professional Experience of Pre-Service Teachers." Teaching Education 23 (1): 71-88. doi:10.1080/10476210.2011.638370

Howells, K., K. Stafford, R. Guiljt, and M. Breadmore. 2017. "The Role of Gratitude in Enhancing the Relationship Between Doctoral Research Students and Their Supervisors. Teaching in Higher Education 22 (6): 621-638. doi:10.1080/13562517.2016.1273212

Hsieh, H. F., and S. E. Shannon. 2005. "Three Approaches to Qualitative Content Analysis." Qualitative Health Research 15 (9): 1277-1288.

Jones, G., J. Milligan, D. Llewellyn, A. Gledhill, and M. I. Johnson. 2017. "Motivational Orientation and Risk Taking in Elite Winter Climbers: A Qualitative Study." International Journal of Sport and Exercise Psychology 15 (1): 25-40. doi:10.1080/1612197x.2015.1069876

Knight, C. J., G. C. D. Little, C. G. Harwood, and K. Goodger. 2016. "Parental Involvement in Elite Junior Slalom Canoeing." Journal of Applied Sport Psychology 28 (2): 234-256. doi:10.1080/10413200.2015.1111273

Lambert, N. M., S. M. Graham, and F. D. Fincham. 2009. "A Prototype Analysis of Gratitude: Varieties of Gratitude Experiences." Personality and Social Psychology Bulletin 35 (9): 1193-1207. doi:10.1177/0146167209338071

Lavelock, C. R., B. J. Griffin, E. L. Worthington, E. G. Benotsch, Y. Lin, C. L. Greer, R. C. Garth, et al. 2016. "A Qualitative Review and Integrative Model of Gratitude and Physical Health." Journal of Psychology and Theology 44: 55-86.

Lodico, M., D. Spaulding, and K. Voegtle. 2010. Methods in Educational Research: From Theory to Practice (2nd ed.). San Francisco, CA: Jossey-Bass.

Longhurst, R. 2003. "Semi-Structured Interviews and Focus Groups." In Key Methods in Geography, edited by N. Clifford, and G. Valentine, 117-132. London: SAGE Publications.

Ma, L. K., R. J. Tunney, and E. Ferguson. 2017. "Does Gratitude Enhance Prosociality? A Meta-Analytic Review." Psychological Bulletin 143 (6): 601-635. doi: http:10.1037/bul0000103

Manela, T. 2016. Negative Feelings of Gratitude." The Journal of Value Inquiry 50 (1): 129140. doi:10.1007/s10790-015-9501-1

Martinsen, M., and J. Sundgot-Borgen. 2013. "Higher Prevalence of Eating Disorders Among Adolescent Elite Athletes Than Controls." Medicine and Science in Sports and Exercise 45: 1188-1197.

McCullough, M. E., R. A. Emmons, and J.-A. Tsang. 2002. "The Grateful Disposition: A Conceptual Empirical Topography." Journal of Personality and Social Psychology 82 (1): 112-127.McConnell, T. 2016 "Gratitude’s Value." Perspectives on Gratitude: An Interdisciplinary Approach, edited by D. Carr, 13-26. London: Routledge.

Miles, M. B., and A. M. Huberman. 1994. Qualitative Data Analysis: An Expanded Sourcebook, 2nd ed. Thousand Oaks, CA: SAGE Publications. 
Morente-Sánchez, J., and M. Zabala. 2013. Doping in Sport: A Review of Elite Athletes' Attitudes, Beliefs, and Knowledge." Sports Medicine 43 (6): 395-411. doi:10.1007/s40279013-0037-x.

Ng, M.-Y., and W.-S. Wong. 2013. "The Differential Effects of Gratitude and Sleep on Psychological Distress in Patients With Chronic Pain." Journal of Health Psychology 18 (2): 263-271.

Nixdorf, I., R. Frank, and J. Beckmann. 2016. "Comparison of Athletes’ Proneness to Depressive Symptoms in Individual and Team Sports: Research on Psychological Mediators in Junior Elite Athletes." Frontiers in Psychology 7: 893. doi:10.3389/fpsyg.2016.00893

O’Connell, B. H., D. O’Shea, and S. Gallagher. 2016. "Enhancing Social Relationships Through Positive Psychology Activities: A Randomised Controlled Trial." The Journal of Positive Psychology, 11 (2): 149-162. doi:10.1080/17439760.2015.1037860

O'Hara, M., C. Carter, P. Dewis, J. Kay, and J. Wainwright, J. 2011. Successful Dissertions. London, England: Continuum International Publishing Group.

Petrocchi, N., and A. Couyoumdjian. 2016. "The Impact of Gratitude on Depression and Anxiety: The Mediating Role of Criticizing, Attacking, and Reassuring the Self." Self and Identity 15 (2): 191-205. doi:10.1080/15298868.2015.1095794

Reardon, C. L., and S. Creado. 2014. "Drug Abuse in Athletes." Substance Abuse and Rehabilitation 5: 95-105. doi: 10.2147/sar.s53784

Roberts, R. 2004. "The Blessings of Gratitude: A Conceptual Analysis." In The Psychology of Gratitude, edited by R. A. Emmons, and M. E. McCullough, 58-79. Oxford, UK: Oxford University Press.

Silverman, D. 2003. "Analyzing Talk and Text." In Collecting and Interpreting Qualitative Materials, edited by N. K. Denzin, and Y. S. Lincoln, 340-362. Thousand Oaks, CA: SAGE Publications.

Smilansky, S. 2002. "Gratitude, Contribution and Ethical Theory." Critical Review of International, Social and Political Philosophy 5 (4): 34-48.

Smith, B. 2018. "Generalizability in Qualitative Research: Misunderstandings. Opportunities and Recommendations for the Sport and Exercise Sciences." Qualitative Research in Sport, Exercise and Health 10 (1): 137-149. doi:10.1080/2159676X.2017.1393221

Smith, J. A., and K. R. McGannon. 2018. "Developing rigor in qualitative research: Problems and opportunities within sport and exercise psychology." International Review of Sport and Exercise Psychology 11 (1): 101-121. doi:10.1080/1750984X.2017.1317357

Strauss, A., and J. Corbin. 1998. Basics of Qualitative Research: Techniques and Procedures for Developing Grounded Theory, 2nd ed. Thousand Oaks, CA: SAGE Publications.

Swann, C., A. Moran, and D. Piggott. 2015. "Defining Elite Athletes: Issues in the Study of Expert Performance in Sport Psychology." Psychology of Sport \& Exercise 16 (1): 3-14. doi:10.1016/ j.psychsport.2014.07.004.

Tsang, J.-A., and S. R. Martin. 2019. "Four Experiments on the Relational Dynamics and Prosocial Consequences of Gratitude." The Journal of Positive Psychology 14 (2): 188-205. doi:10.1080/17439760.2017.1388435

Vieselmeyer, J., J. Holguin, and A. Mezulis. 2016. "The Role of Resilience and Gratitude in Posttraumatic Stress and Growth Following a Campus Shooting." Psychological Trauma 9: 62-69.

Visser, M. 2009. The Gift of Thanks: The Roots and Rituals of Gratitude. New York, NY: Houghton Mifflin Harcourt.

Watkins, P. C., J. Uhder, and S. Pichinevskiy. 2015. "Grateful Recounting Enhances Subjective Well-Being: The Importance of Grateful Processing." The Journal of Positive Psychology 10 (2): 91-98. doi:10.1080/17439760.2014.927909

Wood, A. M., S. Joseph, and J. Maltby. 2009. "Gratitude Predicts Psychological Well-Being Above the Big Five Facets." Personality and Individual Differences 46 (4): 443-447. doi:10.1016/j.paid.2008.11.012 
Yoshimura, S. M., and K. Berzins. 2017. "Grateful Experiences and Expressions: The Role of Gratitude Expressions in the Link Between Gratitude Experiences and Well-Being." Review of Communication 17 (2): 106-118. doi:10.1080/15358593.2017.1293836

Yost-Dubrow, R., and Y. Dunham. 2018. "Evidence for a relationship between trait gratitude and prosocial behaviour." Cognition and Emotion 32 (2): 397-403. doi:10.1080/02699931.2017.1289153 